\title{
Dysregulated Bile Acid Synthesis, Metabolism and Excretion in a High Fat-Cholesterol Diet-Induced Fibrotic Steatohepatitis in Rats
}

\author{
Xiaofang Jia $\cdot$ Hisao Naito $\cdot$ Husna Yetti $\cdot$ Hazuki Tamada $\cdot$ Kazuya Kitamori $\cdot$ \\ Yumi Hayashi · Dong Wang • Yukie Yanagiba · Juncai Wang · Katsumi Ikeda • \\ Yukio Yamori • Tamie Nakajima
}

Received: 20 December 2012/ Accepted: 5 June 2013/Published online: 4 July 2013

(C) The Author(s) 2013. This article is published with open access at Springerlink.com

\begin{abstract}
Background and Aims Cholesterol over-intake is involved in the onset of nonalcoholic steatohepatitis (NASH), and hepatocellular bile acid (BA) accumulation correlates with liver injuries. However, how dietary cholesterol influences cholesterol and BA kinetics in NASH liver remains ambiguous and needs to be clarified.

Methods Molecular markers involved in cholesterol and BA kinetics were investigated at protein and mRNA levels in an already-established stroke-prone spontaneously hypertensive 5/Dmcr rat model with fibrotic steatohepatitis, by feeding a high fat-cholesterol (HFC) diet.
\end{abstract}

Electronic supplementary material The online version of this article (doi:10.1007/s10620-013-2747-1) contains supplementary material, which is available to authorized users.

X. Jia $\cdot$ H. Naito $\cdot$ H. Yetti $\cdot$ H. Tamada $\cdot$ K. Kitamori

Y. Hayashi - D. Wang - Y. Yanagiba - J. Wang - T. Nakajima Department of Occupational and Environmental Health, Nagoya University Graduate School of Medicine, Nagoya 466-8550, Japan

H. Tamada $\cdot$ K. Kitamori

College of Human Life and Environment, Kinjo Gakuin

University, Nagoya 463-8521, Japan

K. Ikeda

School of Pharmacy and Pharmaceutical Sciences, Mukogawa

Women's University, Nishinomiya 663-8179, Japan

Y. Yamori

Institute for World Health Development, Mukogawa Women's

University, Nishinomiya 663-8143, Japan

T. Nakajima $(\bowtie)$

College of Life and Health Sciences, Chubu University,

Kasugai 487-8501, Japan

e-mail: tnasu23@med.nagoya-u.ac.jp
Results Unlike the control diet, the HFC diet deposited cholesterol greatly in rat livers, where 3-hydroxy-3-methylglutaryl CoA reductase, low-density lipoprotein (LDL) receptor and LDL receptor-related protein-1 were expectedly downregulated, especially at 8 and 14 weeks, suggesting that cholesterol synthesis and uptake in response to cholesterol accumulation may not be disorganized. The HFC diet did not upregulate liver X receptor- $\alpha$, conversely, it enhanced classic BA synthesis by upregulating cholesterol $7 \alpha$-hydroxylase but downregulating sterol $12 \alpha$ hydroxylase, and influenced alternative synthesis by downregulating sterol 27-hydroxylase but upregulating oxysterol $7 \alpha$-hydroxylase, mainly at 8 and 14 weeks, indicating that there were different productions of primary BA species. Unexpectedly, no feedback inhibition of BA synthesis by farnesoid X receptor occurred. Additionally, the HFC diet impaired BA detoxification by UDP-glucuronosyltransferase and sulfotransferase $2 \mathrm{~A} 1$, and decreased excretion by bile salt export pump at 8 and 14 weeks, although it induced compensatory export by multidrug resistance-associated protein-3. The disturbed BA detoxification may correlate with suppressed pregnane $\mathrm{X}$ receptor and constitutive androstane receptor.

Conclusions The HFC diet may accumulate BA in rat livers, which influences fibrotic steatohepatitis progression.

Keywords Fibrotic steatohepatitis - High fat-cholesterol diet - Cholesterol and bile acid kinetics - Nuclear receptor

\section{Introduction}

Nonalcoholic steatohepatitis (NASH) is a progressive form of nonalcoholic fatty liver disease (NAFLD), characterized by liver fatty deposition with various degrees of 
inflammation and fibrosis [1]. Although obesity and insulin resistance are considered as pathogenic factors, populations without these risks still could develop NASH $[2,3]$, as lifestyle-related factors such as food intake and food composition may play a role in disease progression [1]. Of these, dietary cholesterol is an important risk factor in the progression of steatosis, inflammatory recruitment and fibrosis in NASH patients [2, 4, 5], and in a wide variety of animal models [6-8]. Interestingly, dietary cholesterol intake has been reported to be abundant in NASH patients without obesity or insulin resistance $[2,3]$.

An animal model with fibrotic steatohepatitis was established by our group by feeding a high fat-cholesterol (HFC) diet to stroke-prone spontaneously hypertensive 5/Dmcr (SHRSP5/Dmcr) rats [9-11]. The most profound feature was severe fibrosis. This model recapitulated histological features of human NASH and exhibited a similar physiological condition to a subgroup of NASH patients without obesity or insulin resistance. In view of the extraordinary cholesterol content (5\% by weight) in the HFC diet, and the hepatic total cholesterol which progressively accumulated as liver disease developed, unlike triglycerides $[9,10]$, the accumulation of cholesterol, rather than triglycerides, may have played a critical role in the progression of fibrotic steatohepatitis in this dietary rat model.

In hepatocytes, cholesterol homeostasis pathways include cholesterol de novo synthesis, uptake in the form of low-density lipoprotein (LDL) and chylomicron remnants, excretion into the blood in the form of very-low-density lipoprotein, excretion and uptake through bile, and catabolism to bile acids (BAs) and their excretion [5]. BA synthesis is initially catalyzed by cholesterol $7 \alpha$-hydroxylase (CYP7A1) in the classic pathway, followed by sterol 12 $\alpha$-hydroxylase (CYP8B1) to form cholic acid and thus control the ratio of cholic acid to chenodeoxycholic acid in the BA pool. The alternative pathway is catalyzed by sterol 27-hydroxylase (CYP27A1), followed by oxysterol $7 \alpha-$ hydroxylase (CYP7B1), and mainly produces chenodeoxycholic acid [12]. BAs are hepatotoxic when their concentrations reach abnormally high levels, because they can cause mitochondria damage, apoptosis or necrosis, and eventually fibrosis and cirrhosis [13]. Indeed, BA levels increased in the liver of NASH patients and significantly correlated with histological inflammation and fibrosis [14]. Taken together, the significance of dietary cholesterol in the development of NASH may be attributable to BAinduced liver injury.

Therefore, we extended our earlier study [11] and used the same liver samples to investigate the abnormalities of cholesterol and BA kinetics in the context of HFC dietinduced fibrotic steatohepatitis in rats.

\section{Materials and Methods}

Diets

A stroke-prone (SP: $20.8 \%$ crude protein, $4.8 \%$ crude lipid, $3.2 \%$ crude fiber, $5.0 \%$ crude ash, $8.0 \%$ moisture, and $58.2 \%$ carbohydrate) diet as a control diet and HFC diet (a mixture of $68 \%$ SP diet, $25 \%$ palm oil, $5 \%$ cholesterol and $2 \%$ cholic acid) were obtained from Funabashi Farm (Chiba, Japan). The components of each diet have been shown elsewhere [9].

Animals

All animal experiments were carried out in accordance with the Guidelines for Animal Experiments of Kinjo Gakuin University Animal Center. Male SHRSP5/Dmcr rats were obtained by mating males and females of the SHRSP5/Dmcr strain with high cholesterol levels $[9,11]$. All rats were housed in a temperature- and light-controlled environment $\left(23 \pm 2{ }^{\circ} \mathrm{C}, 55 \pm 5 \%\right.$ humidity, 12-h light/ dark cycle) with free access to the control chow (SP diet) and tap water.

\section{Experimental Protocols}

At 10 weeks of age, the male offspring were randomly divided into six groups of six rats each and fed with SP or HFC diet for 2,8 and 14 weeks, respectively. After 18-20 $\mathrm{h}$ of fasting from the last feeding of each group, the rats were weighed, anaesthetized by pentobarbital $(70 \mathrm{mg} /$ $\mathrm{kg}$ ) and sacrificed. Blood and livers were harvested. Serum collected by centrifugation of the blood and livers was stored at $-80{ }^{\circ} \mathrm{C}$ until use [11].

\section{Cholesterol Determination}

The levels of serum total cholesterol (TC) and highdensity lipoprotein cholesterol (HDL-C) were determined by S.R.L. Inc. (Tokyo, Japan). Hepatic lipid was extracted as described by Folch et al. [15]. The hepatic TC content was measured using the T-Cho-IE kit (Wako, Osaka, Japan). All of the experiments were repeated at least two times.

UDP-Glucuronosyltransferase (UGT) Activity Assay

UGT activity of 1-naphthol was determined according to the method of Lee et al. [16]. 
Real-Time Quantitative Polymerase Chain Reaction (PCR)

The total RNA was isolated from whole livers using the RNeasy Mini Kit (QIAGEN, Tokyo, Japan). Real-time PCR analysis was performed as described previously [11, 17]. We normalized all of the mRNA expression levels to glyceraldehyde 3-phosphate dehydrogenase (GAPDH) mRNA in the same preparation. The primer sequences are listed in Supplemental Table 1. All assays were repeated three times.

\section{Western Blot Analysis}

A section of each liver was homogenized with three volumes of $0.25 \mathrm{M}$ sucrose-10 mM phosphate buffer ( $\mathrm{pH} 7.4$ ). Nuclear fractions were extracted from portions of frozen liver using the CelLytic ${ }^{\mathrm{TM}}$ NuCLEAR $^{\mathrm{TM}}$ Extraction Kit (SIGMA, Tokyo, Japan) for nuclear receptor measurements. Samples containing the same quantity of protein were subjected to $10 \%$ SDS-PAGE as described previously $[11,17]$. The membranes were incubated with the following primary antibodies: SREBP-2, HMGCoA reductase, LRP1, CYP7A1, CYP8B1, CYP7B1, SULT2A1, BSEP, LXR $\alpha$, FXR and PXR (Santa Cruz Biotechnology, Santa Cruz, CA); LDLR (Cayman Chemical, Ann Arbor, Michigan); CYP27A1 (Proteintech, Chicago, USA); MRP3 (Sigma-Aldrich, St. Louis, MO); and CAR (LifeSpan BioSciences Inc., Seattle, WA). Immunoblotting with GAPDH (Santa Cruz Biotechnology, Santa Cruz, CA) for homogenates and Lamin-B1 (MBL, Nagoya, Japan) for nuclear fraction was performed for loading controls. For the detection of specific proteins, the 1-Step ${ }^{\text {TM }}$ NBT/BCIP (Pierce Biotechnology, Rockford, IL, USA) or ECL Western Blotting Detection Reagent (GE Healthcare, Buckinghamshire, UK) was used. The densities of each band were calculated by CS Analyzer software (Rise \& ATTO Corporation). All assays were repeated three times.

\section{Statistical Analysis}

Quantitative data are expressed as mean \pm standard deviation. The data were initially tested for homogeneity of variances. If the variances were heterogeneous, a logarithm or square root transformation was performed before the analysis. Group differences at the same study period were determined using Student's $t$ test. Differences among three study periods in the SP or HFC group were evaluated using one-way ANOVA followed by Tukey post hoc test. All of the analyses were performed using the SPSS software.
$P$ values less than 0.05 were considered statistically significant.

\section{Results}

Changes in Serum and Hepatic Cholesterol Levels During Development of Fibrotic Steatohepatitis

As previously reported, hepatomegaly occurred in HFC-fed rats, while liver weight remained normal in SP-fed rats during the entire study. Following prolonged HFC-diet feeding, steatohepatitis that developed after 2 weeks of dietary intake evolved to steatohepatitis with moderate fibrosis at 8 weeks, and even severe bridging fibrosis at 14 weeks [11]. Further analysis showed that serum TC levels progressively increased in rats fed either SP or HFC diet during the whole study; moreover, the level in HFCfed rats was higher than in SP-fed rats $(P<0.05$ for all comparisons at each period), especially at 14 weeks when the peak value was 23-fold greater (Fig. 1a, $t=14.875$, $P=0.018$ ). Although serum HDL-C was lower in rats fed the HFC diet at 2 weeks compared with SP-fed rats $(t=77.110, P<0.001)$, it gradually elevated and was significantly greater at 14 weeks (Fig. 1b, $t=12.361$, $P<0.05$ ). The HFC diet (which contains $5 \%$ cholesterol) increased TC content in liver about 57- and 64-fold at 2 and 8 weeks, respectively $(P<0.001$ for all comparisons relative to the SP diet). The HFC diet also induced a maximal accumulation (89-fold) at 14 weeks $(t=2980.703$, $P<0.001$ ), when steatohepatitis with severe fibrosis was established (Fig. 1c).

De Novo Cholesterol Synthesis and Hepatic Uptake Pathway Are Suppressed in HFC-Fed Rats

Although HFC diet feeding had no influence on the sterol regulatory element-binding protein-2 (SREBP-2) mRNA or the nuclear mature protein level (Fig. 2a, b), compared to SP-fed rats, rats fed the HFC-diet exhibited significant decreases in 3-hydroxy-3-methylglutaryl coenzyme A (HMG-CoA) reductase and LDL receptor (LDLR) at mRNA and protein levels, as well as decreases in LDL receptor-related protein-1 (LRP1) protein $(P<0.05$ for all comparisons). Moreover, HMG-CoA reductase, LDLR and LRP1 protein expressions were significantly different among three time-points in HFC-fed rats $\left(F_{(2,15)}=8.318\right.$, $P=0.004 ; F_{(2,15)}=10.676, P=0.001 ; F_{(2,15)}=7.832$, $P=0.005$, respectively), the protein levels of these enzymes were much lower at 8 and/or 14 weeks compared to those at 2 weeks $(P<0.01)$. Additionally, we determined the microsomal triglyceride transfer protein 

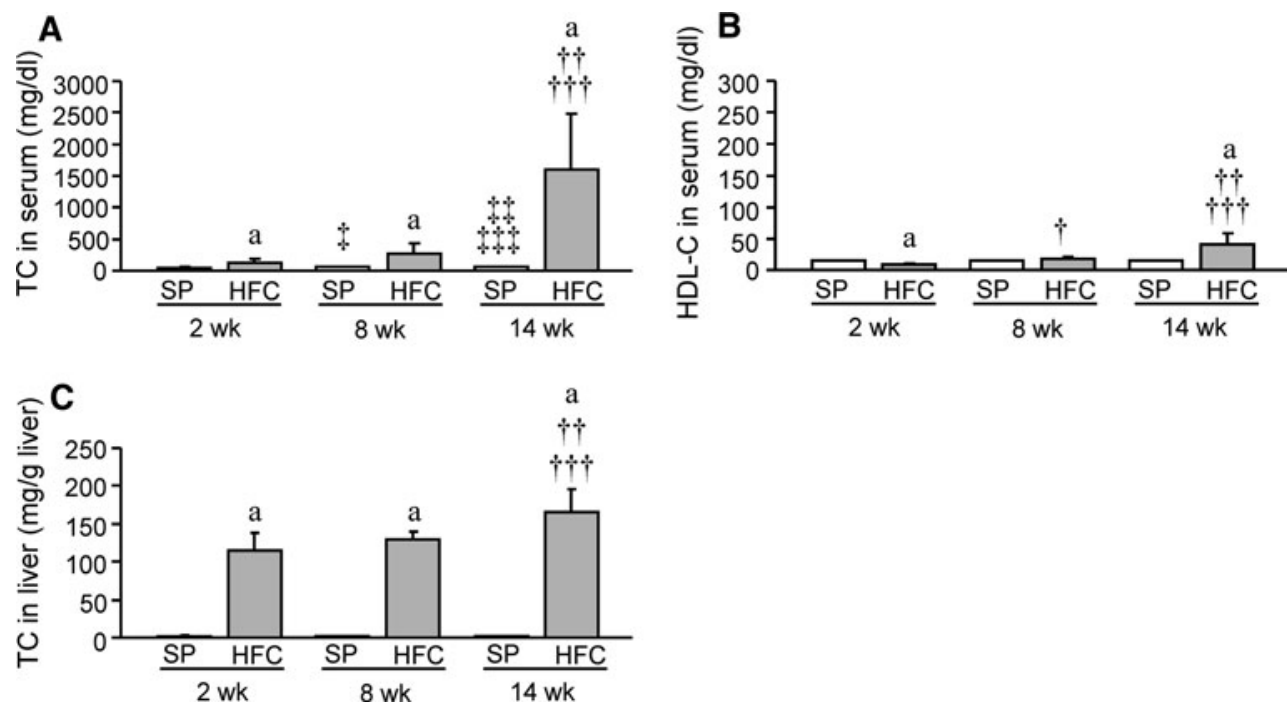

Fig. 1 Cholesterol levels in serum and liver. Serum TC (a) and HDLC (b) levels, and hepatic TC content (c) in rats fed SP or HFC diet for 2,8 and 14 weeks, respectively. $n=6 /$ group. ${ }^{a} P<0.05$ between SP and HFC groups at the same period; $\$, \phi,+\neq, \dagger$ and $\$+,+\dagger \dagger$ means significance $(P<0.05)$ when 2 - vs. 8 -weeks, 2 - vs. 14-weeks, and 8-

vs. 14-weeks, respectively, in the SP and HFC groups. Data of TC levels in serum and liver in rats fed SP and HFC diets were used elsewhere [9]. $H D L-C$ high-density lipoprotein cholesterol, $H F C$ high fat-cholesterol, $S P$ stroke-prone, $T C$ total cholesterol
Fig. 2 Cholesterol biosynthesis and hepatic uptake from blood. a Real-time quantitative PCR of genes involved in hepatic cholesterol synthesis and uptake, including SREBP-2, HMG-CoA reductase, LDLR and LRP1. b Western blot analysis and representative images of SREBP-2, HMGCoA reductase, LDLR and LRP1. $n=6$ /group. ${ }^{\text {a }} P<0.05$ between SP and HFC groups at the same period; ${ }^{\dagger \dagger \dagger}$ means significance $(P<0.05)$ when 2- vs. 8-weeks and 2- vs. 14-weeks, respectively, in the HFC group. $H F C$ high fatcholesterol, $H M G$ - $C O A$ 3-hydroxy-3-methylglutaryl CoA, $L D L R$ low-density lipoprotein receptor, $L R P 1$ lowdensity lipoprotein receptorrelated protein-1, $S P$ strokeprone, SREBP-2 sterol regulatory element-binding protein-2

\section{A mRNA}

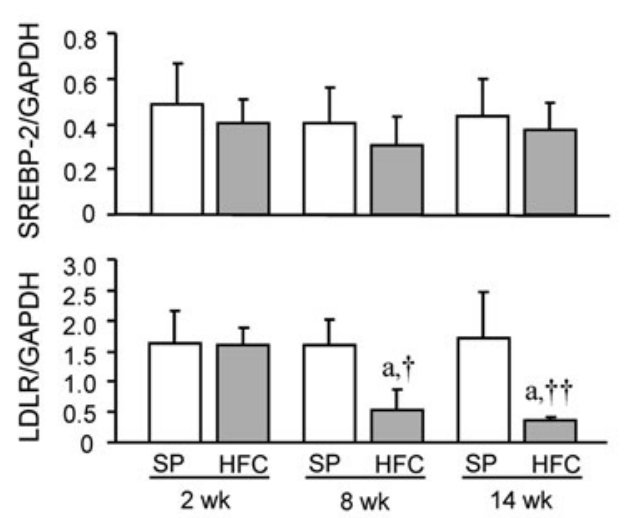

\section{B Protein}
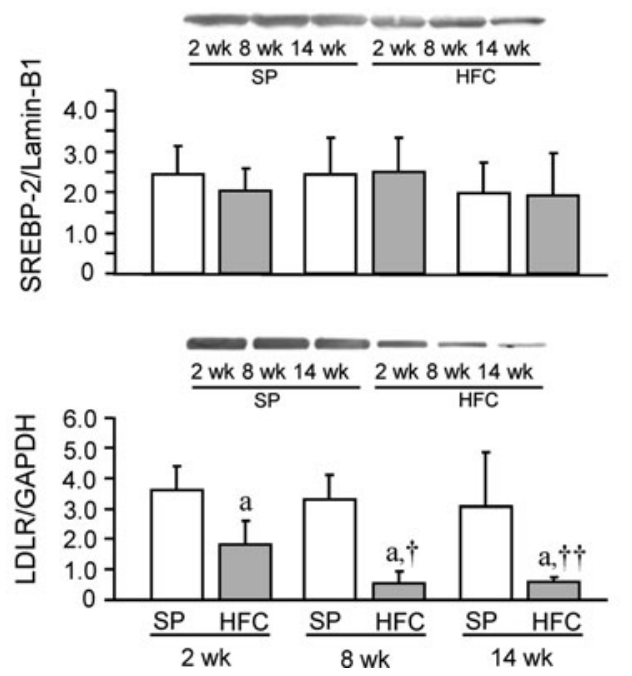
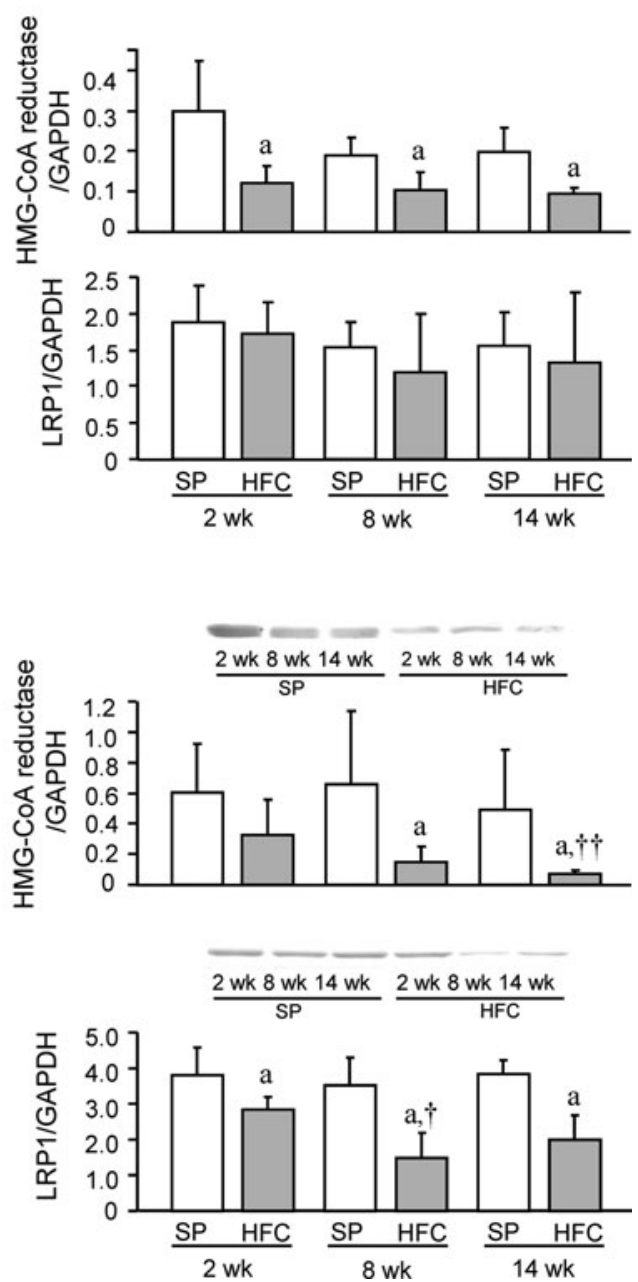
responsible for cholesterol excretion into the blood, but no significant changes were found (data not shown).

BA Synthesis, Export and Transformation Are Dysregulated in HFC-Fed Rats

HFC diet-fed rats exhibited higher CYP7A1 mRNA at 8 weeks $(t=7.724, P=0.038)$ but lower CYP27A1 mRNA at $2(t=21.616, P=0.001)$ and $14(t=14.637$, $P=0.003)$ weeks compared to their SP-fed rat counterparts (Fig. 3). Unlike SP-fed rats, suppressed expression of bile salt export pump (BSEP), a canalicular transporter, at the mRNA level was observed in HFC-fed rats ( $t=46.512, P<0.001 ; t=10.466, P=0.009$ at 8 and 14 weeks, respectively), where BSEP expression was downregulated after 2 weeks $\left(F_{(2,15)}=31.554, \quad P<\right.$ 0.001). Conversely, basolateral transporter multidrug resistance-associated protein-3 (MRP3) was significantly induced at the mRNA level at $8(t=27.889, P<0.001)$ and $14(t=204.226, P<0.001)$ weeks. Additionally, the HFC diet inhibited sulfotransferase 2A1 (SULT2A1) mRNA during the entire study $(t=7.007, P=0.024$; $t=26.765 ; P<0.001 ; t=50.328, P<0.001$, respectively, relative to the SP diet), and inhibited UGT1A1 mRNA at $8(t=4.960, P=0.049)$ and $14(t=12.297$, $P=0.006)$ weeks compared to the SP control diet.

The HFC diet significantly stimulated CYP7A1 protein at $8(t=6.763, P=0.026)$ and $14(t=9.352, P=0.012)$ weeks as opposed to the SP diet (Fig. 4), while CYP8B1
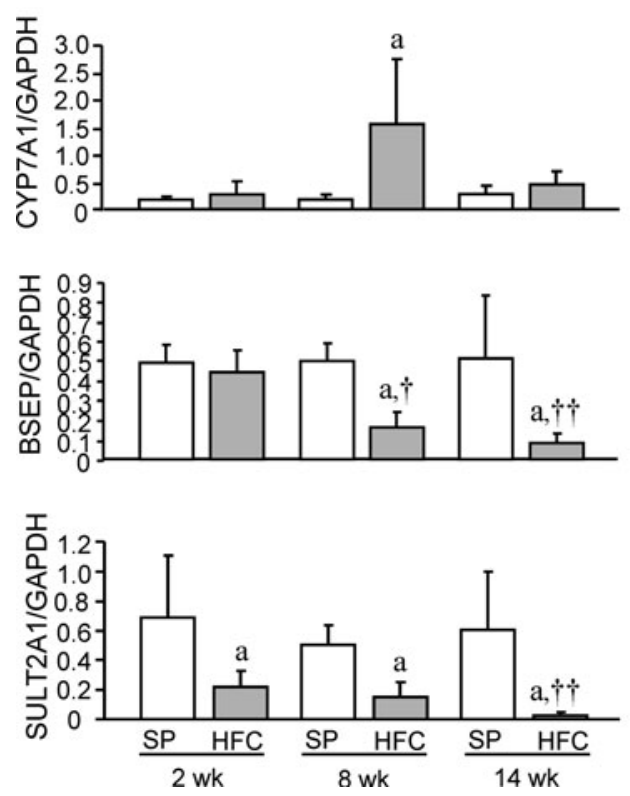

Fig. 3 mRNA levels of markers in BA synthesis, transport and transformation, including CYP7A1, CYP27A1, BSEP, MRP3, SULT2A1 and UGT1A1. $n=6$ /group. ${ }^{a} P<0.05$ between SP and HFC groups at the same period; ${ }^{\dagger}, \dagger^{\dagger}, \dagger^{\dagger}$ means significance $(P<0.05)$ at 2- vs. 8-weeks, 2- vs. 14-weeks and 8-vs. 14-weeks, respectively, protein was highly suppressed over the entire study $(t=44.078, \quad P<0.001 ; \quad t=13.732, \quad P=0.004 ; \quad t=$ 48.898, $P<0.001$, respectively). In the alternative pathway, CYP27A1 was significantly decreased in HFC-fed rats at 14 weeks ( $t=18.648, P=0.002)$, compared to SPfed rats. Conversely, CYP7B1 was highly increased in rats fed an HFC diet for 8 and 14 weeks $(t=20.244$, $P=0.001 ; t=13.836, P=0.004$, respectively). Compared to SP-fed rats, suppressed expression of BSEP at the protein level was observed in HFC-fed rats $(t=7.883$, $P=0.019 ; t=22.759, P=0.001 ; t=35.964, P<0.001$ at each time-point, respectively), where BSEP expression was downregulated from 2 to 14 weeks $\left(F_{(2,15)}=4.566\right.$, $P=0.028)$. Similar to BSEP, expression of another canalicular transporter, MRP2, also reduced in HFC-fed rats (data not shown). Conversely, MRP3 protein was significantly induced at 8 and 14 weeks $(P<0.001$ for all comparisons relative to SP-fed rats). Additionally, the HFC diet highly suppressed SULT2A1 protein at the endpoint $(t=20.117, P=0.001)$, and UGT activity of 1-naphthol at 8 and 14 weeks $(t=154.837, P<0.001 ; t=131.578$, $P<0.001$, respectively), compared to the SP diet.

Nuclear Regulators of BA Homeostasis Are Disturbed in HFC-Fed Rats

BA homeostasis is regulated to a large extent at the transcription level, via nuclear receptors that play a key role in BA synthesis, detoxification and transport systems. We
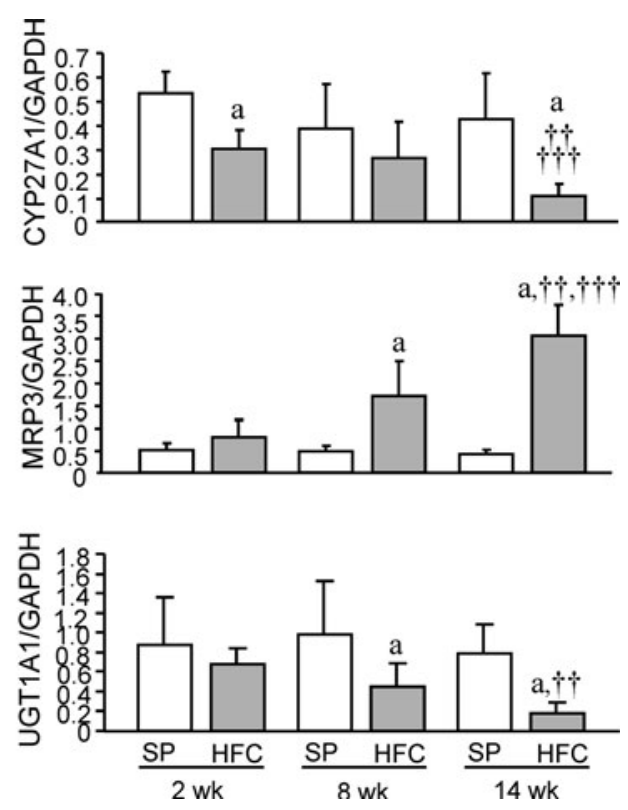

in the HFC group. $B A$ bile acid, $B S E P$ bile salt export pump, $C Y P 7 A 1$ cholesterol $7 \alpha$-hydroxylase, CYP27A1 sterol 27-hydroxylase, HFC high fat-cholesterol, $M R P 3$ multidrug resistance-associated protein-3, $S P$ stroke-prone, SULT2A1 sulfotransferase 2A1, UGT1A1 UDPglucuronosyltransferase $1 \mathrm{~A} 1$ 

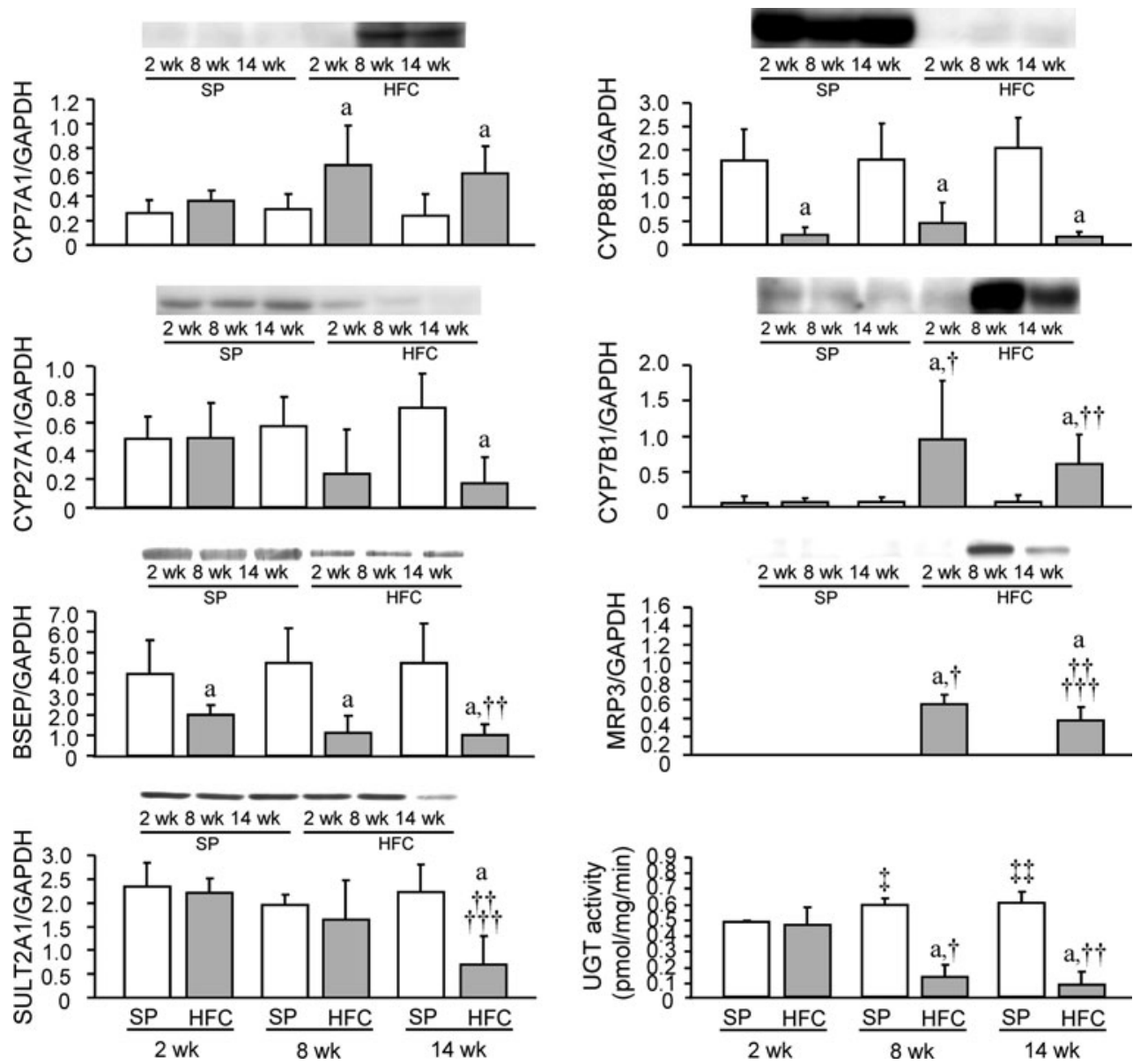

Fig. 4 Western blot analysis and corresponding images of markers involved in BA synthesis, transport and transformation, including CYP7A1, CYP8B1, CYP27A1, CYP7B1, BSEP, MRP3 and SULT2A1. UGT activity of 1-naphthol was also shown. $n=6 /$ group. ${ }^{\mathrm{a}} P<0.05$ between SP and HFC groups at the same period; +, + means significance $(P<0.05)$ when 2 - vs. 8 -weeks and 2 - vs. 14-weeks, respectively, in the SP group; ${ }^{\dagger, \dagger,+\dagger \dagger}$ means significance

measured some members of the nuclear receptors, and unexpectedly, expression of liver X receptor- $\alpha(\mathrm{LXR} \alpha)$ was downregulated in response to an HFC diet during the study. Moreover, LXR $\alpha$ mRNA of the HFC group was lower than that of the SP group at each period (Fig. 5a, $t=6.217, P=0.032 ; t=11.623, P=0.007 ; t=147$. $416, P<0.001$, respectively), as was the protein level at 14 weeks (Fig. $5 b, t=11.986, P=0.006$ ). HFC-diet feeding also significantly decreased mRNA levels of the farnesoid X receptor (FXR) $(t=43.002, \quad P<0.001$; $t=11.124, P=0.008 ; t=237.603, P<0.001$ at each time-point, respectively) and its target gene small heterodimer partner (SHP) $(t=13.280, P=0.005 ; t=50.377$, $P<0.001$ at 8 and 14 weeks, respectively), and tended to decrease the protein level of FXR during the entire study. The alterations in the expression of pregnane $\mathrm{X}$ receptor (PXR) were closely similar to those of LXR $\alpha$. Unlike

$(P<0.05)$ when 2 - vs. 8-weeks, 2 - vs. 14 -weeks and 8-vs. 14-weeks, respectively, in the HFC group. $B A$ bile acid, $B S E P$ bile salt export pump, CYP7A1 cholesterol 7 $\alpha$-hydroxylase, CYP27A1 sterol 27-hydroxylase, $C Y P 8 B 1$ sterol $12 \alpha$-hydroxylase, $C Y P 7 B 1$ oxysterol $7 \alpha$-hydroxylase, $H F C$ high fat-cholesterol, MRP3 multidrug resistance-associated protein-3, SP stroke-prone, SULT2A1 sulfotransferase 2A1, UGT UDP-glucuronosyltransferase

SP-fed rats, rats fed the HFC diet had suppressed constitutive androstane receptor (CAR) mRNA at each period $(t=9.935, \quad P=0.010 ; \quad t=68.371, \quad P<0.001 ; \quad t=$ 52.071, $P<0.001$, respectively) and downregulated CAR protein at $2(t=7.209, P=0.023)$ and $14(t=9.576$, $P=0.011)$ weeks.

\section{Discussion}

We previously reported that the HFC diet time-dependently induced lipid and inflammatory cell accumulations, hepatocyte ballooning, necrosis and fibrosis in the liver of SHRSP5/Dmcr rats, although these rats were not obese or insulin resistant [9-11]. The lipid accumulation in the liver might have resulted from cholesterol rather than triglycerides, because the level of the latter time-dependently 
Fig. 5 Nuclear regulators of BA homeostasis. a Real-time quantitative PCR of $\mathrm{LXR} \alpha$,

FXR and its target gene SHP, PXR and CAR. b Hepatic protein levels of $\mathrm{LXR} \alpha, \mathrm{FXR}$, PXR and CAR determined by western blot. $n=6 /$ group.

${ }^{\mathrm{a}} P<0.05$ between SP and HFC groups at the same period; +. + means significance $(P<0.05)$ when 2 - vs. 8 -weeks and 2- vs. 14-weeks, respectively, in the SP group; $\dagger,+, \dagger+\dagger$ means significance $(P<0.05)$ when 2 - vs. 8-weeks, 2 - vs. 14-weeks and 8- vs. 14-weeks, respectively, in the HFC group. $B A$ bile acid, $C A R$ constitutive androstane receptor, $F X R$ farnesoid $\mathrm{X}$ receptor, $H F C$ high fat-cholesterol, $L X R \propto$ liver $\mathrm{X}$ receptor- $\alpha, P X R$ pregnane $\mathrm{X}$ receptor, $S H P$ small heterodimer partner, $S P$ strokeprone

\section{A mRNA}
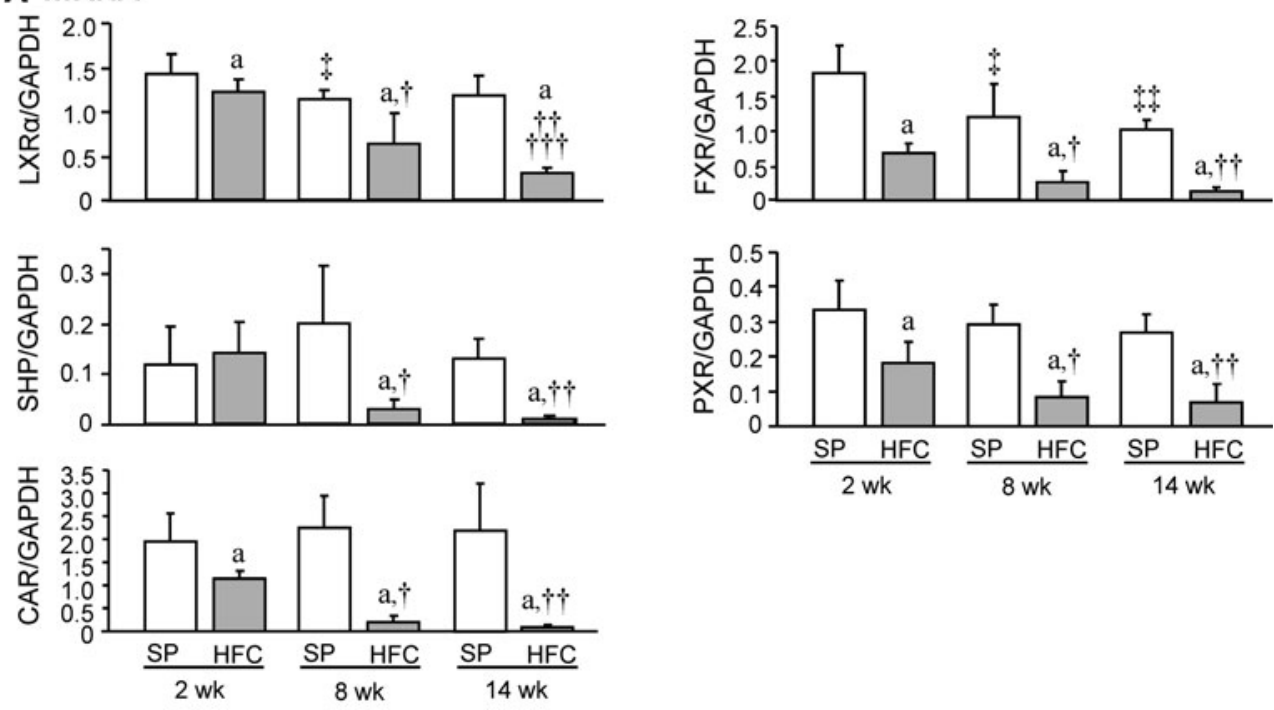

B Protein
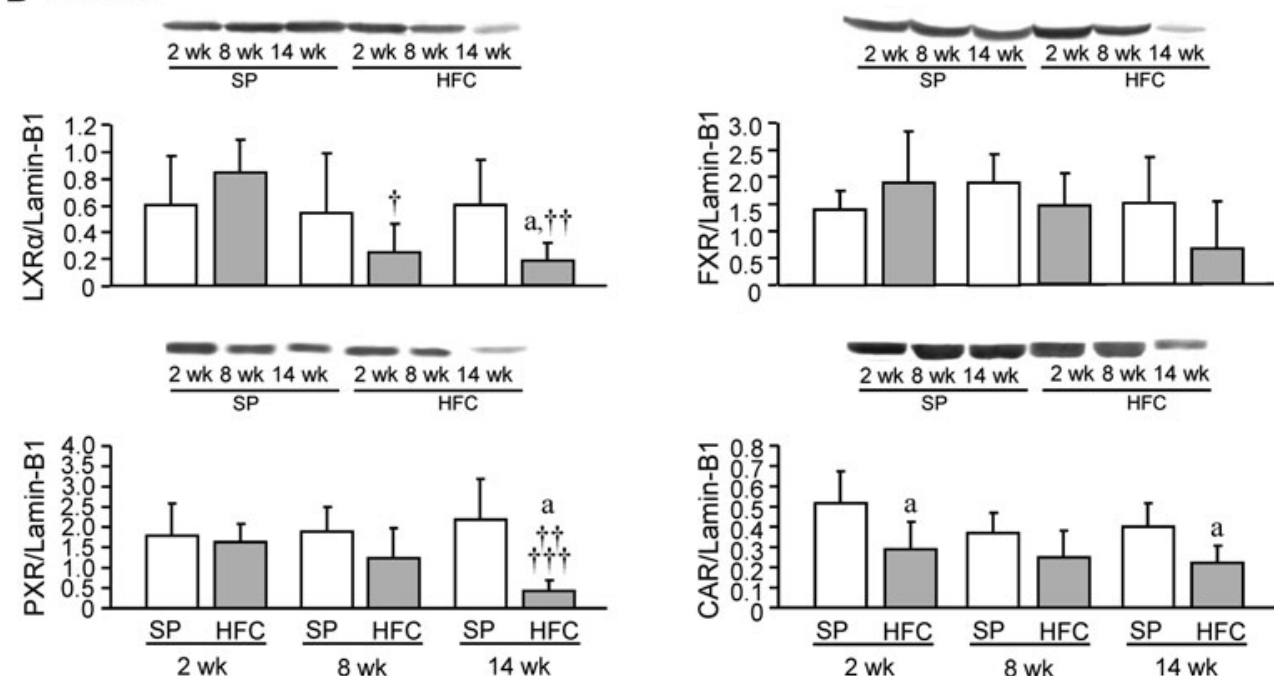

decreased [11]. Thus, the major lipotoxic molecule promoting the pathogenesis of fibrotic steatohepatitis in this model might have been derived from cholesterol.

Feedback regulation of cholesterol biosynthesis mainly occurs in the liver and is exerted primarily on the HMGCoA reductase that catalyzes the rate-limiting step of cholesterol biosynthesis [18]. Similar to previous reports on feedback inhibition by dietary cholesterol [19, 20], hepatic HMG-CoA reductase was down-regulated at 8 and 14 weeks to compensate for the increased absorption of dietary cholesterol. However, SREBP-2, a well-known transcription factor for cholesterol homeostasis, did not change, indicating that not only SREBP-2 but also many additional factors (hormones, cytokines, etc.) might be required for complete control of lipid metabolism [21]. LDLR and LRP1 are members of the LDLR family and contribute to controlling serum cholesterol levels by removing cholesterol-contained lipoproteins from the bloodstream [22]. Dietary cholesterol (1\%) increased hepatic LDLR and had no effect on the plasma cholesterol concentration in Wistar rats, but the effects were opposite in Sprague-Dawley rats [23]. We observed a downregulation of both LDLR and LRP1 in SHRSP5/Dmcr rats fed a $5 \%$ cholesterol diet, and eventually hypercholesterolemia occurred. Such disagreements with previous reports [23] may derive from differences in dietary cholesterol level, strain, and liver condition. Together, cholesterol synthesis and uptake were suppressed as expected in response to cholesterol accumulation in liver with fibrotic steatohepatitis, indicating that these pathways may not be disorganized during the development of steatoheptitis.

We next focused on how dietary cholesterol induced BA accumulation, because it may contribute to understanding the pathogenesis of fibrotic steatoheptitis. Hepatic 
biotransformation of cholesterol into BAs constitutes the major pathway of cholesterol catabolism. Increased conversion into BAs via upregulation of microsomal CYP7A1 in response to cholesterol challenge was confirmed in rats [24, 25], but CYP8B1 did not change [25]. CYP27A1 was induced in the high-cholesterol $(1 \%)$ diet group of rats, but not in mice $[25,26]$, and stayed upregulated in rabbits with a $2 \%$ cholesterol diet intake, regardless of the BA pool size [27]. As expected, in response to HFC diet-induced hepatic cholesterol accumulation in our model, CYP7A1 protein was upregulated over 2-fold, but CYP8B1 was suppressed in the classic synthesis pathway. And while CYP27A1 was downregulated, CYP7B1 was induced in the alternative pathway, especially at 8 and 14 weeks, when fibrotic steatohepatitis occurred. This suggested that an increased BA production, especially chenodeoxycholic acid rather than cholic acid, may be involved in fibrogenesis. Our further study indeed identified an increment in hepatic chenodeoxycholic acid rather than in cholic acid, especially during these periods, by analysis of BA profiles (Jia X et al., manuscript in submission). Downregulations of CYP27A1 and CYP8B1 were also observed in NAFLD patients [28] and the mouse model of NASH [7], but no upregulations of CYP7A1 and CYP7B1 were observed. CYP27A1 is a mitochondrial protein and its downregulation may reflect mitochondrial injury that is present in NASH development [28].

BA homeostasis is under the regulation of nuclear receptors. $\mathrm{LXR} \alpha$ is a sterol sensor, and increased intercellular cholesterol drives the production of oxysterols, which are agonists of $\operatorname{LXR} \alpha$ [29]. $\mathrm{LXR} \alpha$ regulates cholesterol catabolism by acting as a positive regulator of CYP7A1, and mediates fatty acid synthesis by inducing SREBP-1c expression. However, in combination with our earlier study [11], neither the cholesterol-LXR $\alpha-C Y P 7 A 1$ nor the cholesterol-LXR $\alpha$-SREBP-1c signaling pathway was stimulated in our model, unlike with NAFLD patients $[5,29]$. The reason for this apparent discrepancy requires further investigation. FXR is proposed to be a nuclear BA receptor and directly or indirectly (via SHP) inhibits CYP7A1. FXR was not activated in rats fed $2 \%$ cholesterol for 1 week, where CYP7A1 was stimulated and the BA pool size was stable [30]. Likewise, CYP7A1 protein was continuously induced from 8 to 14 weeks in rats fed a $5 \%$ cholesterol-contained HFC diet, but nuclear FXR was not. Nevertheless, hepatic chenodeoxycholic acid species, the most potent FXR ligand [31], was found to increase in HFC-fed rats throughout the study (Jia X et al., manuscript in submission). Therefore, the failure of FXR upregulation by BAs may suggest that there was a disturbed feedback regulation of BA synthesis in our model.

Biliary compounds such as BAs and bilirubin are cytotoxic when present in abnormally high concentrations.
Both glucuronidation and sulfation become pivotal eliminating pathways in cholestasis. They transform hydrophobic, toxic substrates into more hydrophilic, less-toxic derivatives for biliary and urinary excretion, although glycine and taurine conjugation are primarily involved in the process [32, 33]. The most profound changes in the pathways of BA turnover in this dietary model were disturbed UGT-catalyzed glucuronidation, blunted SULT2A1catalyzed sulfation, and impaired BSEP-mediated canalicular export to bile duct, especially observed at 8 and 14 weeks, when fibrosis progressed. All of these disorders, together with upregulations of BA synthetic enzymes, revealed hepatic accumulation of toxic biliary constituents, as evidenced by a direct analysis of BA profiles in liver (Jia $\mathrm{X}$ et al., manuscript in submission). Consequently, toxic biliary compound overload in liver may have contributed to the pathogenesis of liver damage in this model, especially severe fibrosis, as confirmed by the correlation analysis between specific BAs and parameters of liver injury (Jia X et al., manuscript in submission). HFC-diet feeding also induced MRP3-regulated basolateral excretion, an important alternative spillover route during BA overload, which was in agreement with the changes under a cholestatic condition [34]. These changes allow BA elimination from liver to the blood, but are not sufficient for a complete detoxification of the liver cells.

FXR, PXR and CAR protect against hepatotoxicity of BAs in a complementary manner by regulating genes involved in BA transport and detoxification, such as BSEP, UGT and SULT [33, 35]. Simultaneous suppression of nuclear FXR, PXR and CAR occurred with the development of fibrotic steatoheptitis, especially at 14 weeks. On one hand, the downward shift of nuclear FXR expression in HFC-fed rats may correlate with the suppressed transcription of BSEP; on the other hand, downregulated PXR and CAR may result in reduced UGT activity and SULT2A1 protein. PXR and CAR also regulate the transport of MRP3 [33]; however, regardless of the two nuclear regulators, MRP3 was spontaneously enhanced as a compensatory efflux pathway in response to reduced BA transport during cholestasis [34]. Therefore, upregulated MRP3 may not have been dependent on the downregulation of these nuclear receptors in our model.

Finally, we shed insight into the inconsistencies of gene expression in mRNA and protein levels, especially at some particular time points, such as LDLR (2 weeks), LRP1 (each time-point), LXR $\alpha$ ( 2 and 8 weeks), FXR (each timepoint), PXR (2 and 8 weeks) and CAR ( 8 weeks). Our study analyzed mRNA and protein levels in triplicate and observed similar results. Therefore, we excluded experimental errors. Gene expression is a highly complex process that comprises many steps, from transcription to protein degradation. Following transcription, the pre-mRNA is 


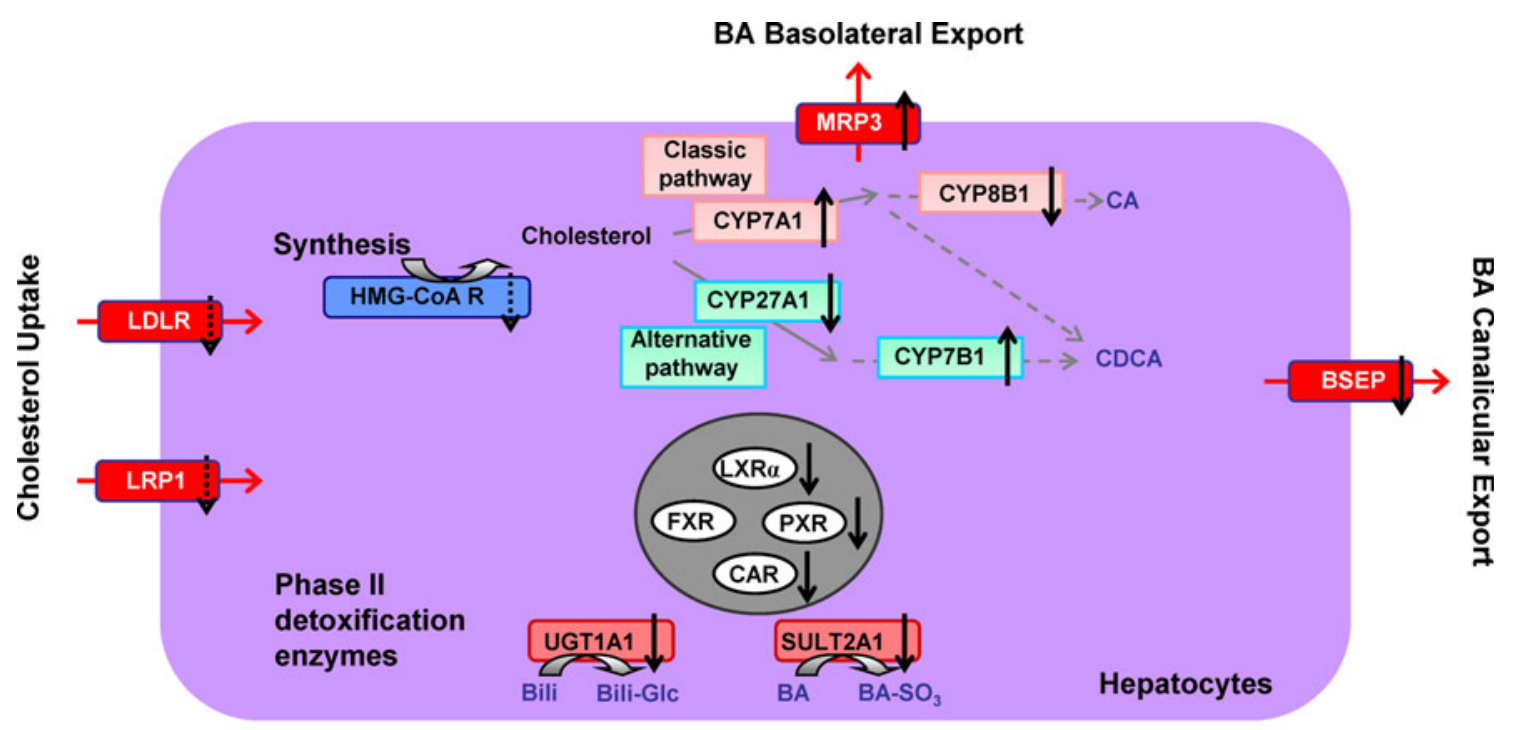

BA Detoxification

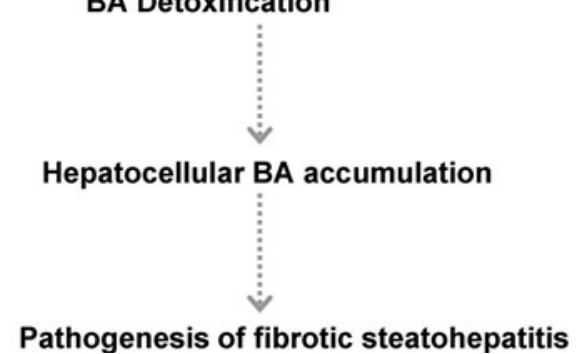

Fig. 6 Hepatic cholesterol synthesis and catabolism enzymes, uptake receptors, BA transport systems, phase II detoxification enzymes, and nuclear receptors investigated in this study. HMG-CoA R is the ratelimiting enzyme of cholesterol biosynthesis. CYP7A1 represents the key enzyme for the catabolism of cholesterol to BA in the classic pathway, followed by CYP8B1. The alternative pathway is initially catalyzed by CYP27A1, followed by CYP7B1. LDLR and LRP1 contribute to controlling the serum cholesterol level by removing cholesterol-contained lipoproteins from the bloodstream. Canalicular BA export is largely mediated by BSEP. Alternative BA export is mediated via basolateral MRP3. The phase II conjugation is mediated by UGT1A1 and SULT2A1. Nuclear receptors such as LXR $\alpha$, FXR, PXR and CAR coordinately regulate BA homeostasis. The changes in these markers in the liver of the HFC diet-induced fibrotic steatohepatitis rat model were indicated using arrows. Dotted black

processed and exported from the nucleus to the cytoplasm where additional steps of mRNA maturation take place. The mature mRNA is then either translated, stored, or degraded. Each step in gene expression is under elaborate regulation and different phases of the process are coupled [36]. Thus, the entire gene expression process can be viewed as a more complex network with feedback between coupled regulatory mechanisms. The discrepancy between mRNA and protein levels could be considered as reasonable phenomena. Additionally, the fatty acid type in HFC diet may affect the expression of mRNA for LDLR and LRP1, as fatty acids from the diet delivered to the liver in chylomicron remnants influenced the transcription of arrows denote expected suppression of cholesterol synthesis and uptake from blood after HFC-diet feeding. Solid black arrows denote abnormal alterations observed in this model. Dotted gray arrows denote presumptions from all these findings. $\mathrm{BA}$ bile acid, $\mathrm{BA}-\mathrm{SO}_{3}$ sulfated BA, Bili bilirubin, Bili-Glc glucuronidated bilirubin, BSEP bile salt export pump, $C A$ cholic acid, $C A R$ constitutive androstane receptor, $C D C A$ chenodeoxycholic acid, CYP7A1 cholesterol $7 \alpha-$ hydroxylase, $C Y P 8 B 1$ sterol $12 \alpha$-hydroxylase, CYP27A1 sterol 27-hydroxylase, $C Y P 7 B 1$ oxysterol $7 \alpha$-hydroxylase, $F X R$ farnesoid $\mathrm{X}$ receptor, $H F C$ high fat-cholesterol, $H M G-C o A R$ 3-hydroxy-3methylglutaryl CoA reductase, $L D L R$ low-density lipoprotein receptor, $L R P 1$ low-density lipoprotein receptor-related protein-1, $L X R \alpha$ liver $\mathrm{X}$ receptor- $\alpha, M R P 3$ multidrug resistance-associated protein-3, $P X R$ pregnane $\mathrm{X}$ receptor, SULT2A1 sulfotransferase $2 \mathrm{~A} 1$, UGT1A1 UDP-glucuronosyltransferase 1A1

hepatic genes regulating their uptake, such as LDLR and LRP1 [37]. In the liver of the HFC diet-induced rat model, to maintain cholesterol homeostasis, LDL uptake may have been suppressed by inducing degradation of LDLR and LRP1 via protein ubiquitination [38, 39]. $\mathrm{LXR} \alpha, \mathrm{FXR}$, PXR and CAR have functional connections in the regulation of cholesterol biosynthesis and metabolism to BAs in liver [40]. Their protein expressions tended to decrease in the HFC diet-induced rat model during the whole study, although significance relative to control was limited. Posttranslational modification or trafficking might explain the differences between mRNA and mature protein [41], which warrants further investigation. 
Many NAFLD patients are not obese, and many of them do not exhibit insulin resistance or increased visceral fat volume, which compel us to question what triggers and maintains the disease in non-obese patients [29]. Interestingly, a nutritional assessment of NAFLD patients revealed that dietary cholesterol intake was significantly higher in non-obese patients than in obese ones [3]. Indeed, in animal models, it has been reported that a high-cholesterol diet caused NASH without triggering obesity or insulin resistance [42]. Although we used a $5 \%$ cholesterol-contained HFC diet in the present study, a $2.5 \%$-cholesterol diet could also induce fibrotic steatohepatitis, to a lesser extent, in SHRSP5/Dmcr rats (data not shown), suggesting that cholesterol dose-dependently induced the liver damage. Together, in combination with diverse changes in hepatic triglycerides and cholesterol with liver disease progression [11], cholesterol over-intake and accumulation in liver may be more potential than high fat intake and triglyceride deposition in our non-obese and non-diabetic model.

In conclusion, the alterations in hepatic cholesterol synthesis and catabolism enzymes, uptake receptors, BA transport systems, phase II detoxification enzymes, and nuclear receptors investigated in the liver of an HFC diet-induced fibrotic steatohepatitis rat model are summarized in Fig. 6. Primarily, cholesterol over-intake in this model accumulated cholesterol in rat livers, which further upregulated BA synthetic enzymes, CYP7A1 and CYP7B1, whereas it downregulated BSEP, UGT and SULT2A1, especially at 8 and 14 weeks. These changes may result in BA accumulation in the liver and mediate a transition towards fibrotic steatohepatitis. Therefore, this study suggests that cholesterol overintake may be one of the potent inducers of fibrotic steatohepatitis, which is mainly achieved via hepatotoxicity of BA accumulation rather than a well-established cholesterolLXR $\alpha$-SREBP-1c pathway in humans, although hepatotoxicity of cholesterol accumulation may be considered.

Acknowledgments This work was supported by a Grant-in-Aid for Scientific Research (B23390161) from the Japan Society for the Promotion of Science.

\section{Conflict of interest None.}

Open Access This article is distributed under the terms of the Creative Commons Attribution Noncommercial License which permits any noncommercial use, distribution, and reproduction in any medium, provided the original author(s) and the source are credited.

\section{References}

1. Farrell GC, Larter CZ. Nonalcoholic fatty liver disease: from steatosis to cirrhosis. Hepatology. 2006;43:S99-S112.

2. Musso G, Gambino R, De Michieli F, et al. Dietary habits and their relations to insulin resistance and postprandial lipemia in nonalcoholic steatohepatitis. Hepatology. 2003;37:909-916.
3. Yasutake K, Nakamuta M, Shima Y, et al. Nutritional investigation of non-obese patients with non-alcoholic fatty liver disease: the significance of dietary cholesterol. Scand J Gastroenterol. 2009; 44:471-477.

4. Caballero F, Fernandez A, De Lacy AM, Fernandez-Checa JC, Caballeria J, Garcia-Ruiz C. Enhanced free cholesterol, SREBP-2 and StAR expression in human NASH. $J$ Hepatol. 2009;50: 789-796.

5. Enjoji M, Yasutake K, Kohjima M, Nakamuta M. Nutrition and nonalcoholic fatty liver disease: the significance of cholesterol. Int J Hepatol. 2012;2012:925807.

6. Subramanian S, Goodspeed L, Wang S, et al. Dietary cholesterol exacerbates hepatic steatosis and inflammation in obese LDL receptor-deficient mice. J Lipid Res. 2011;52:1626-1635.

7. Van Rooyen DM, Larter CZ, Haigh WG, et al. Hepatic free cholesterol accumulates in obese, diabetic mice and causes nonalcoholic steatohepatitis. Gastroenterology. 2011;141:1393-1403.

8. Wouters K, van Gorp PJ, Bieghs V, et al. Dietary cholesterol, rather than liver steatosis, leads to hepatic inflammation in hyperlipidemic mouse models of nonalcoholic steatohepatitis. Hepatology. 2008;48:474-486.

9. Kitamori K, Naito H, Tamada H, et al. Development of novel rat model for high-fat and high-cholesterol diet-induced steatohepatitis and severe fibrosis progression in SHRSP5/Dmcr. Environ Health Prev Med. 2012;17:173-182.

10. Moriya T, Kitamori K, Naito H, et al. Simultaneous changes in highfat and high-cholesterol diet-induced steatohepatitis and severe fibrosis and those underlying molecular mechanisms in novel SHRSP5/Dmcr rat. Environ Health Prev Med. 2012;17: 444-456.

11. Jia X, Naito H, Yetti $H$, et al. The modulation of hepatic adenosine triphosphate and inflammation by eicosapentaenoic acid during severe fibrotic progression in the SHRSP5/Dmcr rat model. Life Sci. 2012;90:934-943.

12. Li T, Chiang JY. Bile acid signaling in liver metabolism and diseases. J Lipids. 2012;2012:754067.

13. Hofmann AF. The continuing importance of bile acids in liver and intestinal disease. Arch Intern Med. 1997;159:2647-2658.

14. Aranha MM, Cortez-Pinto H, Costa A, et al. Bile acid levels are increased in the liver of patients with steatohepatitis. Eur $J$ Gastroenterol Hepatol. 2008;20:519-525.

15. Folch J, Lees M, Sloane Stanley GH. A simple method for the isolation and purification of total lipids from animal tissues. $J$ Biol Chem. 1957;226:497-509.

16. Lee $\mathrm{CH}$, Ito Y, Yanagiba Y, et al. Pyrene-induced CYP1A2 and SULT1A1 may be regulated by CAR and not by AhR. Toxicology. 2007;238:147-156.

17. Ramdhan DH, Kamijima M, Wang D, et al. Differential response to trichloroethylene-induced hepatosteatosis in wild-type and PPAR $\alpha$-humanized mice. Environ Health Perspect. 2010;118:1557-1563.

18. Gould RG. Lipid metabolism and atherosclerosis. Am J Med. 1951;11:209-227.

19. Chambers CM, Ness GC. Dietary cholesterol regulates hepatic 3-hydroxy-3-methylglutaryl coenzyme A reductase gene expression in rats primarily at the level of translation. Arch Biochem Biophys. 1998;354:317-322.

20. Lutton C. Dietary cholesterol, membrane cholesterol and cholesterol synthesis. Biochimie. 1991;73:1327-1334.

21. Weber LW, Boll M, Stampfl A. Maintaining cholesterol homeostasis: sterol regulatory element-binding proteins. World $J$ Gastroenterol. 2004;10:3081-3087.

22. Hussain MM, Strickland DK, Bakillah A. The mammalian low-density lipoprotein receptor family. Annu Rev Nutr. 1999;19:141-172.

23. Roach PD, Balasubramaniam S, Hirata F, et al. The low-density lipoprotein receptor and cholesterol synthesis are affected differently 
by dietary cholesterol in the rat. Biochim Biophys Acta. 1993;1170:165-172.

24. Boone LR, Brooks PA, Niesen MI, Ness GC. Mechanism of resistance to dietary cholesterol. J Lipids. 2011;2011:101242.

25. Chen W, Suruga K, Nishimura N, Gouda T, Lam VN, Yokogoshi $\mathrm{H}$. Comparative regulation of major enzymes in bile acid biosynthesis pathway by cholesterol, cholate and taurine in mice and rats. Life Sci. 2005;77:746-757.

26. Schwarz M, Russell DW, Dietschy JM, Turley SD. Alternative pathways of bile acid synthesis in the cholesterol $7 \alpha$-hydroxylase knockout mouse are not upregulated by either cholesterol or cholestyramine feeding. J Lipid Res. 2001;42:1594-1603.

27. $\mathrm{Xu} \mathrm{G}$, Salen G, Shefer $\mathrm{S}$, et al. Increasing dietary cholesterol induces different regulation of classic and alternative bile acid synthesis. J Clin Invest. 1999;103:89-95.

28. Min HK, Kapoor A, Fuchs M, et al. Increased hepatic synthesis and dysregulation of cholesterol metabolism is associated with the severity of nonalcoholic fatty liver disease. Cell Metab. 2012; 15:665-674.

29. Nakamuta M, Fujino T, Yada R, et al. Impact of cholesterol metabolism and the LXRalpha-SREBP-1c pathway on nonalcoholic fatty liver disease. Int J Mol Med. 2009;23:603-608.

30. Xu G, Pan LX, Li H, et al. Dietary cholesterol stimulates CYP7A1 in rats because farnesoid $\mathrm{X}$ receptor is not activated. Am J Physiol Gastrointest Liver Physiol. 2004;286:G730-G735.

31. Parks DJ, Blanchard SG, Bledsoe RK, et al. Bile acids: natural ligands for an orphan nuclear receptor. Science. 1999;284: $1365-1368$.

32. Trottier J, Milkiewicz P, Kaeding J, Verreault M, Barbier O. Coordinate regulation of hepatic bile acid oxidation and conjugation by nuclear receptors. Mol Pharm. 2006;3:212-222.

33. Wagner M, Halilbasic E, Marschall HU, et al. CAR and PXR agonist stimulate hepatic bile acid and billirubin detoxification and elimination pathways in mice. Hepatology. 2005;42:420-430.

34. Takikawa H. Hepatobiliary transport of bile acids and organic anions. J Hepatobiliary Pancreat Surg. 2002;9:443-447.
35. Guo GL, Lambert G, Negishi M, et al. Complementary roles of farnesoid $\mathrm{X}$ receptor, pregnane $\mathrm{X}$ receptor, and constitutive androstane receptor in protection against bile acid toxicity. $J$ Biol Chem. 2003;278:45062-45071.

36. Dahan O, Gingold H, Pilpel Y. Regulatory mechanisms and network couple the different phases of gene expression. Trends Genet. 2011;27:316-322.

37. Zheng X, Rivabene R, Cavallari C, et al. The effects of chylomicron remnants enriched in $n-3$ or $n-6$ polyunsaturated fatty acids on the transcription of genes regulating their uptake and metabolism by the liver: influence of cellular oxidative state. Free Radic Biol Med. 2002;32:1123-1131.

38. Do HT, Tselykh TV, Makela J, et al. Fibroblast growth factor-21 (FGF21) regulates low-density lipoprotein receptor (LDLR) levels in cells via the E3-ubiquitin ligase Mylip/Idol and the Canopy2 (Cnpy2)/Mylip-interacting Saposin-like protein (Msap). $J$ Biol Chem. 2012;287:12602-12611.

39. Cal R, Garcia-Arguinzonis M, Revuelta-Lopez E, et al. Aggregated low-density lipoprotein induces LRP1 stabilization through E3 ubiquitin ligase CHFR downregulation in human vascular smooth muscle cells. Arterioscler Thromb Vasc Biol. 2013;33: 369-377.

40. Handschin C, Meyer UA. Regulatory network of lipid-sensing nuclear receptors: roles for CAR, PXR, LXR, and FXR. Arch Biochem Biophys. 2005;433:387-396.

41. Myohanen TT, Garcia-Horsman JA, Tenorio-Laranga J, et al. Issues about the physiological functions of prolyl oligopeptidase based on its discordant spatial association with substrates and inconsistencies among mRNA, protein levels, and enzymatic activity. J Histochem Cytochem. 2009;57:831-848.

42. Kainuma M, Fujimoto M, Sekiya N, et al. Cholesterol-fed rabbit as a unique model of nonalcoholic, nonobese, no-insulin-resistant fatty liver disease with characteristic fibrosis. $J$ Gastroenterol. 2006;41:971-980. 